\title{
ENDPOINT FOR THE DIV-CURL LEMMA IN HARDY SPACES
}

\author{
ALINE BONAMI, JUSTIN FEUTO, AND SANDRINE GRELLIER
}

\begin{abstract}
We give a div-curl type lemma for the wedge product of closed differential forms on $\mathbb{R}^{n}$ when they have coefficients respectively in a Hardy space and $L^{\infty}$ or a space of $B M O$ type. In this last case, the wedge product belongs to an appropriate Hardy-Orlicz space.
\end{abstract}

\section{INTRODUCTION}

The theory of compensated compactness initiated and developed by L. Tartar [Ta] and F. Murat [M] has been largely studied and extended to various setting. The famous paper of Coifman, Lions, Meyer and Semmes ( [CLMS]) gives an overview of this theory in the context of Hardy spaces in the Euclidean space $\mathbb{R}^{n}(n \geq 1)$. They prove in particular, that, for $\frac{n}{n+1}<p, q<\infty$ such that $\frac{1}{p}+\frac{1}{q}<1+\frac{1}{n}$, when $F$ is a vector field belonging to the Hardy space $\mathcal{H}^{p}\left(\mathbb{R}^{n}, \mathbb{R}^{n}\right)$ with curl $F=0$ and $G$ is a vector field belonging to $\mathcal{H}^{q}\left(\mathbb{R}^{n}, \mathbb{R}^{n}\right)$ with $\operatorname{div} G=0$, then the scalar product $F \cdot G$ can be given a meaning as a distribution of $\mathcal{H}^{r}\left(\mathbb{R}^{n}\right)$ with

$$
\|F \cdot G\|_{\mathcal{H}^{r}\left(\mathbb{R}^{n}\right)} \leq C\|F\|_{\mathcal{H}^{p}\left(\mathbb{R}^{n}, \mathbb{R}^{n}\right)}\|G\|_{\mathcal{H}^{q}\left(\mathbb{R}^{n}, \mathbb{R}^{n}\right)}
$$

where $\frac{1}{r}=\frac{1}{p}+\frac{1}{q}$.

The endpoint $\frac{n}{n+1}$ is related to cancellation properties of Hardy spaces: bounded functions with compact support and zero mean do not belong to $\mathcal{H}^{\frac{n}{n+1}}$ unless their moments of order one are zero, a property that the scalar product $F . G$ does not have in general.

We shall consider here the endpoint $q=\infty$. Let us first start by some description of what is known. Auscher, Russ and Tchamitchian remarked in ART that, for $p=1$, one has, under the same assumptions of being respectively curl free and divergence free,

$$
\|F \cdot G\|_{\mathcal{H}^{1}\left(\mathbb{R}^{n}\right)} \leq C\|F\|_{\mathcal{H}^{1}\left(\mathbb{R}^{n}, \mathbb{R}^{n}\right)}\|G\|_{L^{\infty}\left(\mathbb{R}^{n}, \mathbb{R}^{n}\right)} .
$$

1991 Mathematics Subject Classification. 42B30 (58A10). forms.

Key words and phrases. Hardy-spaces, Hardy-Orlicz spaces, div-curl lemma, differential

The authors are partially supported by the project ANR AHPI number ANR-07-BLAN0247-01. 
In fact it is easy to see that the proof given in [CLMS] is also valid for $q=\infty$. They give in [ART another proof, which has its own interest and has helped us in our generalization to $B M O$. Remark that the scalar product does not make sense in general when $F$ is in $\mathcal{H}^{p}\left(\mathbb{R}^{n}, \mathbb{R}^{n}\right)$ for $p<1$, so that one can only write a priori estimates, such as the following one.

Theorem 1.1. Let $\frac{n}{n+1}<p \leq 1$. If $F \in \mathcal{H}^{p}\left(\mathbb{R}^{n}, \mathbb{R}^{n}\right)$ is integrable and such that curl $F=0$ and if $G \in L^{\infty}\left(\mathbb{R}^{n}, \mathbb{R}^{n}\right)$ with $\operatorname{div} G=0$, then there exists a constant $C$, independent of $F$ and $G$, such that

$$
\|F \cdot G\|_{\mathcal{H}^{p}\left(\mathbb{R}^{n}\right)} \leq C\|F\|_{\mathcal{H}^{p}\left(\mathbb{R}^{n}, \mathbb{R}^{n}\right)}\|G\|_{L^{\infty}\left(\mathbb{R}^{n}, \mathbb{R}^{n}\right)} .
$$

This a priori estimate allows to give a meaning to $F \cdot G$ in the distribution sense. There is no hope to give such a meaning for general products $f g$, with $f \in \mathcal{H}^{p}\left(\mathbb{R}^{n}\right)$ and $g \in L^{\infty}$. It is proved in $[\mathrm{BF}]$ that this is possible when $g$ is in the inhomogeneous Lipschitz space $\Lambda_{\alpha}\left(\mathbb{R}^{n}\right)$, with $\alpha=n\left(\frac{1}{p}-1\right)$. Moreover, one has

$$
f g \in L^{1}\left(\mathbb{R}^{n}\right)+\mathcal{H}^{p}\left(\mathbb{R}^{n}\right)
$$

for $f$ in $\mathcal{H}^{p}$ (with $p<1$ ) and $g$ in $\Lambda_{n\left(\frac{1}{p}-1\right)}$. So, cancellation properties of the scalar product of curl free and divergence free vector fields allow to get rid of the integrable part and to weaken the assumptions from Lipschitz to bounded.

We will show in Section 4 that this generalizes to wedge products of closed forms. Remark that end-point estimates would imply all other ones by interpolation if we could interpolate between $\mathcal{H}^{p}$ spaces of closed forms. Indeed, for instance, the generalization to closed forms allows to have (3) when assumptions on the two factors are exchanged: $F$ is bounded and $G$ is in $\mathcal{H}^{p}\left(\mathbb{R}^{n}, \mathbb{R}^{n}\right)$. Unfortunately, one does not know whether one can interpolate: while there is a bounded projection onto closed forms in $\mathcal{H}^{p}$ for $p<\infty$, it is not the case for $p=\infty$.

The core of this paper concerns div-curl lemmas (and their extensions for the wedge product of closed forms) when the assumption to be bounded is weakened into an assumption of type $B M O$. Products of functions in $\mathcal{H}^{1}$ and $B M O$ have been considered by Bonami, Iwaniec, Jones and Zinsmeister in [BIJZ]. Such products make sense as distributions, and can be written as the sum of an integrable function and a function in a weighted Hardy-Orlicz space. In order to have a div-curl lemma in this context, we make some restriction for one of the two factors. Recall that $\mathfrak{b m o}:=\mathfrak{b} \mathfrak{m o}\left(\mathbb{R}^{n}\right)$ is the set of locally integrable functions $b$ satisfying

$$
\sup _{|B| \leq 1}\left(\frac{1}{|B|} \int_{B}\left|b-b_{B}\right| d x\right)<\infty \quad \text { and } \sup _{|B| \geq 1}\left(\frac{1}{|B|} \int_{B}|b| d x\right)<\infty
$$


with $B$ varying among all balls of $\mathbb{R}^{n}$ and $|B|$ denoting the measure of the ball $B$. The sum of the two finite quantities will be denoted by $\|b\|_{\mathfrak{b m o}}$. Then $\mathfrak{b} \mathfrak{m o}$ is well-known to be the dual space of the localized version of the Hardy space, which we note $\mathfrak{h}^{1}\left(\mathbb{R}^{n}\right)$, see [G]. To be more precise, for $f \in \mathcal{H}^{1}\left(\mathbb{R}^{n}\right)$ and $g \in \mathfrak{b} \mathfrak{m o}$, we define the product (in the distribution sense) $f g$ as the distribution whose action on the Schwartz function $\varphi \in \mathcal{S}\left(\mathbb{R}^{n}\right)$ is given by

$$
\langle f g, \varphi\rangle:=\langle\varphi g, f\rangle \text {, }
$$

where the second bracket stands for the duality bracket between $\mathcal{H}^{1}$ and $B M O$. It is then proved in [BIJZ] that

$$
f g \in L^{1}\left(\mathbb{R}^{n}\right)+\mathcal{H}_{\omega}^{\Phi}\left(\mathbb{R}^{n}\right) .
$$

Here $\mathcal{H}_{\omega}^{\Phi}\left(\mathbb{R}^{n}\right)$ is the weighted Hardy-Orlicz space related to the Orlicz function

$$
\Phi(t):=\frac{t}{\log (e+t)}
$$

and with weight $\omega(x):=(\log (e+|x|))^{-1}$. This extends immediately to vectorvalued functions. In the next theorem, we prove that there is no $L^{1}$ term in the context of the div-curl lemma.

Theorem 1.2. Let $F \in \mathcal{H}^{1}\left(\mathbb{R}^{n}, \mathbb{R}^{n}\right)$ with $\operatorname{curl} F=0$ and $G \in \mathfrak{b m o}\left(\mathbb{R}^{n}, \mathbb{R}^{n}\right)$ with $\operatorname{div} G=0$. Then there exists some constant $C$, independent of $F$ and $G$, such that

$$
\|F \cdot G\|_{\mathcal{H}_{\omega}^{\Phi}\left(\mathbb{R}^{n}\right)} \leq C\|F\|_{\mathcal{H}^{1}\left(\mathbb{R}^{n}, \mathbb{R}^{n}\right)}\|G\|_{\mathfrak{b m o}\left(\mathbb{R}^{n}, \mathbb{R}^{n}\right)} .
$$

The theorem is also valid for $G \in B M O$, but for local Hardy spaces $\mathfrak{h}^{1}$ and $\mathfrak{h}_{\omega}^{\Phi}$ instead of $\mathcal{H}^{1}$ and $\mathcal{H}_{\omega}^{\Phi}$. We do not know whether it is valid without this restriction on $F$ or $F \cdot G$. There is an $\mathcal{H}^{p}$ version of this theorem, for $p<1$, which we give also. Note that div-curl have been developed in the context of local Hardy spaces by Dafni [D].

These results can be compared to what can be said on products of holomorphic functions, for which analogous estimates are elementary and have a weak converse, see [BG].

To simplify notations, we restricted to vector fields in the introduction, but we shall write below these results in the context of the wedge product of closed differential forms. Indeed, recall that a divergence free vector field can be identified with an $(n-1)$-closed differential form, while a curl free vector field identifies with a 1- closed form, their scalar product being given by the wedge product of these two forms. The usual div-curl lemma has been extended to wedge products of closed differential forms by Lou and Mc Intosh [LM1, LM2] when $\frac{1}{p}+\frac{1}{q}=1$, with both $p$ and $q$ finite. We will do it in general.

Our paper is organized as follows. We recall basic results about classical Hardy spaces in the second section. We define an appropriate grand maximal 
function to characterize $\mathcal{H}^{p}\left(\mathbb{R}^{n}\right)$, which has been introduced in ART. In Section 3, after recalling some basic facts about differential forms, we give the analogous of the previous grand maximal function characterization in this context. In Section 4 we give the whole range of the div-curl Lemma for closed forms. Section 5 is devoted to assumptions of type $B M O$.

Throughout this paper, $C$ denotes constants that are independent of the functions involved, with values which may differ from line to line. For two quantities $A$ and $B$, the notation $A \sim B$ means that there exist two positive constants $C_{1}$ and $C_{2}$ such that $C_{1} A \leq B \leq C_{2} A$. If $E$ is a measurable subset of $\mathbb{R}^{n}$, then $|E|$ stands for its Lebesgue measure.

\section{Some Basic facts about Classical Hardy SPACES}

We fix $\varphi \in \mathcal{S}\left(\mathbb{R}^{n}\right)$ having integral 1 and support in the unit ball $\mathbb{B}=$ $\left\{x \in \mathbb{R}^{n}:|x|<1\right\}$. For $f \in \mathcal{S}^{\prime}\left(\mathbb{R}^{n}\right)$ and $x \in \mathbb{R}^{n}$, we put

$$
(f * \varphi)(x):=\langle f, \varphi(x-\cdot)\rangle,
$$

and define the maximal function $\mathcal{M} f=\mathcal{M}_{\varphi} f$ by

$$
\mathcal{M} f(x):=\sup _{t>0}\left|\left(f * \varphi_{t}\right)(x)\right|,
$$

where $\varphi_{t}(x)=t^{-n} \varphi\left(t^{-1} x\right)$.

For $p>0$, a tempered distribution $f$ is said to belong to the Hardy space $\mathcal{H}^{p}\left(\mathbb{R}^{n}\right)$ if

$$
\|f\|_{\mathcal{H}^{p}\left(\mathbb{R}^{n}\right)}:=\left(\int_{\mathbb{R}^{n}} \mathcal{M}_{\varphi} f(x)^{p} d x\right)^{\frac{1}{p}}=\left\|\mathcal{M}_{\varphi} f\right\|_{L^{p}}
$$

is finite. It is well known that, up to equivalence of corresponding norms, the space $\mathcal{H}^{p}\left(\mathbb{R}^{n}\right)$ does not depend on the choice of the function $\varphi$. So, in the sequel, we shall use the notation $\mathcal{M} f$ instead of $\mathcal{M}_{\varphi} f$.

For $\frac{n}{n+1}<p \leq 1$, an $\mathcal{H}^{p}$-atom (related to the ball $B$ ) is a bounded function $a$ supported in $B$ and satisfying the following conditions

$$
\|a\|_{L^{\infty}} \leq|B|^{-\frac{1}{p}} \text { and } \int_{\mathbb{R}^{n}} a(x) d x=0 .
$$

The atomic decomposition of $\mathcal{H}^{p}$ states that a temperate distribution $f$ belongs to $\mathcal{H}^{p}$ if and only if there exist a sequence $\left(a_{j}\right)$ of $\mathcal{H}^{p}$-atoms and a sequence $\left(\lambda_{j}\right)$ of scalars such that

$$
f=\sum_{j=1}^{\infty} \lambda_{j} a_{j} \quad \text { and } \quad \sum_{j=1}^{\infty}\left|\lambda_{j}\right|^{p}<\infty
$$


where the first sum is assumed to converge in the sense of distributions. Moreover, $f$ is the limit of the partial sums in $\mathcal{H}^{p}$, and $\|f\|_{\mathcal{H}^{p}}$ is equivalent to the infimum, taken over all such decomposition of $f$, of the quantities $\left(\sum_{j=1}^{\infty}\left|\lambda_{j}\right|^{p}\right)^{\frac{1}{p}}$.

We refer to $\mathrm{St}$ ] for background on Hardy spaces.

For the purpose of our main results, we are going to define an appropriate grand maximal function, which induces on $\mathcal{H}^{p}$ a semi-norm equivalent to the previous one.

Let $q>n$. For $x \in \mathbb{R}^{n}$, we denote by $\mathcal{F}_{x}^{q}$, the set of all $\psi \in W^{1, q}\left(\mathbb{R}^{n}\right)$ supported in some ball $B(x, r)$ centered at $x$ with radius $r>0$ which satisfy

$$
\|\psi\|_{L^{q}\left(\mathbb{R}^{n}\right)}+r\|\nabla \psi\|_{L^{q}\left(\mathbb{R}^{n}\right)} \leq\left|B_{(x, r)}\right|^{-\frac{1}{q^{\prime}}},
$$

where $\frac{1}{q}+\frac{1}{q^{\prime}}=1$. Here $W^{1, q}\left(\mathbb{R}^{n}\right)$ denotes the Sobolev space of functions in $L^{q}$ with derivatives in $L^{q}$. Since $q>n$, the Sobolev theorem guarantees that the test functions are bounded, which allows to give the following definition.

For $f \in L_{l o c}^{1}\left(\mathbb{R}^{n}\right)$, and $x \in \mathbb{R}^{n}$, put

$$
\mathcal{M}_{q} f(x):=\sup _{\psi \in \mathcal{F}_{x}^{q}}\left|\int_{\mathbb{R}^{n}} f \psi\right| .
$$

The following lemma is classical, but we give its proof for completeness.

Lemma 2.1. Let $f$ be a locally integrable function on $\mathbb{R}^{n}$.

(i) There exists a constant $C$ not depending on $f$, such that

$$
\mathcal{M} f \leq C \mathcal{M}_{\infty} f
$$

(ii) For $\frac{n}{n+1}<p \leq 1$ and $\frac{1}{q}<\frac{n+1}{n}-\frac{1}{p}$

$$
\left\|\mathcal{M}_{q} f\right\|_{L^{p}\left(\mathbb{R}^{n}\right)} \sim\|f\|_{\mathcal{H}^{p}\left(\mathbb{R}^{n}\right)} .
$$

Proof. Let $f \in L_{l o c}^{1}\left(\mathbb{R}^{n}\right)$. To prove (i), it is sufficient to see that, for $\varphi$ the test function used in the definition of Hardy space, there exists some constant $c$ such that, for all $x \in \mathbb{R}^{n}$ and $t>0$, the function $\varphi_{x, t}(y):=c \varphi_{t}(x-y)$ belongs to $\mathcal{F}_{x}^{\infty}$. One can choose $c=\left(\|\varphi\|_{L^{\infty}\left(\mathbb{R}^{n}\right)}+\|\nabla \varphi\|_{L^{\infty}}\right)^{-1}$.

Let us now prove (ii). It is sufficient to consider $q<\infty$ and the inequality

$$
\left\|\mathcal{M}_{q} f\right\|_{L^{p}} \leq C\|f\|_{\mathcal{H}^{p}}
$$

since

$$
\mathcal{M} f \leq C \mathcal{M}_{\infty} f \leq C \mathcal{M}_{q} f .
$$

By sub-linearity of the maximal operator $\mathcal{M}_{q}$, it is sufficient to prove a uniform estimate for atoms,

$$
\left\|\mathcal{M}_{q} a\right\|_{L^{p}} \leq C
$$


for some uniform constant $C$. Indeed, once we have this, we conclude for $f=\sum \lambda_{j} a_{j}$ that

$$
\left\|\mathcal{M}_{q} f\right\|_{L^{p}} \leq\left(\sum\left|\lambda_{j}\right|^{p}\left\|\mathcal{M}_{q} a\right\|_{L^{p}}^{p}\right)^{1 / p} \leq C\left(\sum\left|\lambda_{j}\right|^{p}\right)^{1 / p}
$$

So let us prove (21). Without loss of generality, using invariance by translation and dilation, we may assume that $a$ is a function with zero mean, supported by the unit ball $\mathbb{B}$ centered at 0 , and bounded by 1 . We prove that there exists $\varepsilon>0$ depending on $q$ such that

$$
\left|\mathcal{M}_{q} a(x)\right| \leq C(1+|x|)^{-n-1+\varepsilon} .
$$

By assumption on $\psi \in \mathcal{F}_{x}^{q}$, using Hölder's Inequality, we find that $\|\psi\|_{1} \leq 1$. So $\mathcal{M}_{q} a$ is bounded by 1 , and it is sufficient to prove that, for $\psi \in \mathcal{F}_{x}^{q}$,

$$
\left|\int_{\mathbb{B}} \psi a\right| \leq C|x|^{-n-1+\varepsilon}
$$

for $|x| \geq 2$. Moreover, in this range of $x$, we may restrict to functions $\psi$ supported in $B(x, r)$ with $r>|x| / 2$, so that $\|\nabla \psi\|_{q} \leq C|x|^{-\frac{n}{q^{\prime}}-1}$.

Since $a$ has mean zero,

$$
\left|\int_{\mathbb{B}} \psi a\right|=\left|\int_{\mathbb{B}}\left(\psi-\psi_{\mathbb{B}}\right) a\right| \leq C\|\nabla \psi\|_{q} .
$$

We have used Poincaré Inequality for the last inequality. The condition on $q$ is required for $|x|^{-p\left(\frac{n}{q^{\prime}}+1\right)}$ to be integrable at infinity.

This discussion extends to local Hardy spaces, that we define now. We first define the truncated version of the maximal function, namely

$$
\mathcal{M}_{\varphi}^{(1)} f(x):=\sup _{0<t<1}\left|\left(f * \varphi_{t}\right)(x)\right| .
$$

A tempered distribution $f$ is said to belong to the space $\mathfrak{h}^{p}\left(\mathbb{R}^{n}\right)$ if

$$
\|f\|_{\mathfrak{h}^{p}\left(\mathbb{R}^{n}\right)}:=\left(\int_{\mathbb{R}^{n}} \mathcal{M}_{\varphi}^{(1)} f(x)^{p} d x\right)^{\frac{1}{p}}<\infty .
$$

The atomic decomposition holds for local Hardy spaces, with only atoms associated to balls of radius less than 1 satisfying the moment condition, see $G$. The previous lemma is valid in the context of $\mathfrak{h}^{p}\left(\mathbb{R}^{n}\right)$, with $\mathcal{M}^{(1)}$ in place of $\mathcal{M}$. 


\section{HARDY SPACES OF DIFFERENTIAL FORMS}

Let us first fix notations and recall standard properties. Let $\mathbb{R}^{n}$ be the Euclidean space equipped with its standard orthonormal basis $\mathcal{B}=\left\{e_{1}, \ldots, e_{n}\right\}$, and let $\mathcal{B}^{*}=\left\{e^{1}, \ldots, e^{n}\right\}$ be its dual basis.

For $\ell \in\{1, \ldots, n\}$, denote by $\Lambda^{\ell}$ the space of $\ell$-linear alternating forms, which consists in linear combinations of exterior products

$$
e^{I}=e^{i_{1}} \wedge \ldots \wedge e^{i_{\ell}}
$$

where $I=\left(i_{1}, \ldots, i_{\ell}\right)$ is any $\ell$-tuple. The standard basis of $\Lambda^{\ell}$ is $\left\{e^{I}\right\}$ where $I$ is an ordered $\ell$-tuple, $1 \leq i_{1}<\ldots<i_{\ell} \leq n$. For $\alpha=\sum_{I} \alpha_{I} e^{I}$ and $\beta=\sum_{I} \beta_{I} e^{I}$ in $\Lambda^{\ell}$, we define the inner product of $\alpha$ and $\beta$ as follows

$$
\langle\alpha, \beta\rangle:=\sum \alpha_{I} \beta_{I}
$$

where the summation is taken over all ordered $\ell$-tuples.

The Hodge operator is the linear operator $*: \Lambda^{\ell} \rightarrow \Lambda^{n-\ell}$ defined by

$$
\alpha \wedge * \beta=\langle\alpha, \beta\rangle e^{1} \wedge \ldots \wedge e^{n}
$$

for all $\alpha, \beta \in \Lambda^{\ell}$.

An $\ell$-form on $\mathbb{R}^{n}$ is defined as a function $u: \mathbb{R}^{n} \rightarrow \Lambda^{\ell}$ which may be written as

$$
u=\sum_{I} u_{I} e^{I}
$$

where the $u_{I}$ 's are (real-valued) functions on $\mathbb{R}^{n}$ and all the I's are of length $\ell$.

Definition 3.1. Let $\Omega \subset \mathbb{R}^{n}$ be an open set, $\ell$ a positive integer as above and $\mathcal{E}(\Omega)$ a normed space of functions $f: \Omega \rightarrow \mathbb{R}$ equipped with the norm $\|f\|_{\mathcal{E}(\Omega)}$. We say that an $\ell$-form $\omega=\sum_{I} \omega_{I} e^{I}$ belongs to $\mathcal{E}\left(\Omega, \Lambda^{\ell}\right)$ if $\omega_{I} \in \mathcal{E}(\Omega)$ for all ordered $\ell$-tuples $I$, and we pose

$$
\|\omega\|_{\mathcal{E}\left(\Omega, \Lambda^{\ell}\right)}:=\sum_{I}\left\|\omega_{I}\right\|_{\mathcal{E}(\Omega)}
$$

Let $d: \mathcal{D}^{\prime}\left(\Omega, \Lambda^{\ell-1}\right) \rightarrow \mathcal{D}^{\prime}\left(\Omega, \Lambda^{\ell}\right)$ denote the exterior derivative operator given by

$$
d \omega=\sum_{k, I} \partial_{k} \omega_{I} e^{k} \wedge e^{I}
$$

where $\partial_{k} \omega_{I}$ is the partial derivative with respect to the $k$-th variable. The Hodge operator $\delta: \Lambda^{\ell} \rightarrow \Lambda^{\ell-1}$ defined by $\delta=(-1)^{n(n-\ell)} * d *$ is the formal adjoint of $d$ in the sense that if $\alpha \in \mathcal{C}^{\infty}\left(\Omega, \Lambda^{\ell}\right)$ and $\beta \in \mathcal{C}^{\infty}\left(\Omega, \Lambda^{\ell+1}\right)$, then

$$
\int_{\Omega}\langle\alpha, \delta \beta\rangle=-\int_{\Omega}\langle d \alpha, \beta\rangle,
$$


provided that one of these forms has compact support. We also define the Laplacian

$$
\Delta_{\ell}=d \delta+\delta d: \mathcal{D}^{\prime}\left(\mathbb{R}^{n}, \Lambda^{\ell}\right) \rightarrow \mathcal{D}^{\prime}\left(\mathbb{R}^{n}, \Lambda^{\ell}\right)
$$

and a simple calculation shows that for $\omega=\sum_{I} \omega_{I} e^{I} \in W^{2, p}\left(\mathbb{R}^{n}, \Lambda^{\ell}\right)$ with $1 \leq p \leq \infty$

$$
\Delta_{\ell} \omega=\sum_{I} \Delta \omega_{I} e^{I}
$$

where $\Delta \omega_{I}$ is the usual Laplacian on functions.

For $f=\sum_{I} f_{I} e^{I} \in \mathcal{D}^{\prime}\left(\mathbb{R}^{n}, \Lambda^{\ell}\right)$, we put

$$
\partial_{j} f:=\sum_{I} \partial_{j} f_{I} e^{I}
$$

Definition 3.2. Let $\ell \in\{1, \ldots, n-1\}$, and $\frac{n}{n+1}<p \leq 1$. The Hardy space of closed $\ell$-forms is defined as

$$
\mathcal{H}_{d}^{p}\left(\mathbb{R}^{n}, \Lambda^{\ell}\right):=\left\{f \in \mathcal{H}^{p}\left(\mathbb{R}^{n}, \Lambda^{\ell}\right): \quad d f=0\right\}
$$

endowed with the norm of $\mathcal{H}^{p}\left(\mathbb{R}^{n}, \Lambda^{\ell}\right)$.

Recall that all closed $\ell$-forms are exact, that is, there exists some $g \in$ $\mathcal{D}^{\prime}\left(\mathbb{R}^{n}, \Lambda^{\ell-1}\right)$ such that $f=d g$.

We will need the analogue of the previous scalar characterizations of Hardy spaces.

For $1 \leq q \leq \infty$, we first define, for $f \in L_{l o c}^{1}\left(\mathbb{R}^{n}, \Lambda^{\ell}\right)$, the grand maximal function $\overrightarrow{\mathcal{M}}_{q} f$ as follows.

$$
\overrightarrow{\mathcal{M}}_{q} f(x):=\sup _{\Phi \in \overrightarrow{\mathcal{F}}_{x}^{q}}\left|\int_{\mathbb{R}^{n}} f \wedge \Phi\right|,
$$

where $\overrightarrow{\mathcal{F}}_{x}^{q}$ denote the set of all $\Phi \in W^{1, q}\left(\mathbb{R}^{n}, \Lambda^{n-\ell}\right)$ for which there exists $r>0$ such that $\Phi$ is supported in the ball $B_{(x, r)}$ and satisfies

$$
(*) \quad\|\Phi\|_{L^{q}\left(\mathbb{R}^{n}, \Lambda^{n-\ell}\right)}+r\|\nabla \Phi\|_{L^{q}\left(\mathbb{R}^{n}, \Lambda^{n-\ell}\right)} \leq\left|B_{(x, r)}\right|^{-\frac{1}{q^{\prime}}} .
$$

The next lemma is a direct consequence of Lemma 2.1 and the fact that for $f=\sum_{I} f_{I} e^{I} \in \mathcal{H}^{p}\left(\mathbb{R}^{n}, \Lambda^{\ell}\right)$ and any positive integer $k$,

$$
\overrightarrow{\mathcal{M}}_{q} f \leq \sum_{I} \mathcal{M}_{q} f_{I}
$$

Lemma 3.3. Let $\frac{n}{n+1}<p \leq 1$ and $\frac{1}{q}<\frac{n+1}{n}-\frac{1}{p}$. There exists a constant $C$ such that, for all $f \in L_{l o c}^{1}\left(\mathbb{R}^{n}, \Lambda^{\ell}\right)$,

$$
\left\|\overrightarrow{\mathcal{M}}_{q} f\right\|_{L^{p}\left(\mathbb{R}^{n}\right)} \leq C\|f\|_{\mathcal{H}^{p}\left(\mathbb{R}^{n}, \Lambda^{\ell}\right)} .
$$


We need a weaker version of this grand maximal function, denoted by $\overrightarrow{\mathcal{M}}_{q, d} f$, which is adapted to Hardy spaces of closed forms. We define

$$
\overrightarrow{\mathcal{M}}_{q, d} f(x):=\sup _{\Phi \in \overrightarrow{\mathcal{F}}_{x, d}^{q}}\left|\int_{\mathbb{R}^{n}} f \wedge \Phi\right|,
$$

where $\overrightarrow{\mathcal{F}}_{x, d}^{q}$ denote the set of $\Phi \in L^{\infty}\left(\mathbb{R}^{n}, \Lambda^{n-\ell}\right)$ supported in some ball $B(x, r)$ satisfying

$$
(* *) \quad\|\Phi\|_{L^{q}\left(\mathbb{R}^{n}, \Lambda^{n-\ell}\right)}+r\|d \Phi\|_{L^{q}\left(\mathbb{R}^{n}, \Lambda^{n-\ell+1}\right)} \leq\left|B_{(x, r)}\right|^{-\frac{1}{q^{\prime}}} .
$$

Lemma 3.4. Let $q>n$ and $1 \leq \ell \leq n-1$. For all $f \in L_{l o c}^{1}\left(\mathbb{R}^{n}, \Lambda^{\ell}\right)$, the following inequality holds

$$
\overrightarrow{\mathcal{M}}_{q} f \leq \overrightarrow{\mathcal{M}}_{q, d} f
$$

Moreover, if $f$ is a closed form, then

$$
\overrightarrow{\mathcal{M}}_{q, d} f \leq C \overrightarrow{\mathcal{M}}_{q} f
$$

for some uniform constant $C$.

Proof. Let $\Phi=\sum_{I} \Phi_{I} e^{I} \in \mathcal{C}^{\infty}\left(\mathbb{R}^{n}, \Lambda^{n-\ell}\right)$. It follows from the fact that $d \Phi=$ $\sum_{I, j} \partial_{j} \Phi_{I} e^{j} \wedge e^{I}$, that

$$
\|d \Phi\|_{L^{q\left(\mathbb{R}^{n}, \Lambda^{n-\ell+1}\right)}} \leq \sum_{I, j}\left\|\partial_{j} \Phi_{I}\right\|_{L^{q\left(\mathbb{R}^{n}\right)}} \leq\|\nabla \Phi\|_{L^{q\left(\mathbb{R}^{n}, \Lambda^{n-\ell}\right)}} .
$$

Thus, for all $x \in \mathbb{R}^{n}$, we have $\overrightarrow{\mathcal{F}}_{x}^{q} \subset \overrightarrow{\mathcal{F}}_{x, d}^{q}$ so that (39) follows from the definition of the maximal functions $\overrightarrow{\mathcal{M}}_{q} f$ and $\overrightarrow{\mathcal{M}}_{q, d} f$.

Assume now that $f$ is a locally integrable closed form. Remark first that, for $\phi$ and $\psi$ bounded compactly supported such that $d \psi=d \phi$, we have

$$
\int f \wedge \phi=\int f \wedge \psi
$$

Indeed, we can assume by regularization that $f$ is a smooth function on some open set containing the supports of $\phi$ and $\psi$. Moreover, $f$ may be written as $d g$, with $g$ a smooth function on this open set. So the equality follows from integration by parts.

Now, let $x \in \mathbb{R}^{n}$ and $\Phi=\sum_{I} \Phi_{I} e^{I} \in \overrightarrow{\mathcal{F}}_{x, d}^{q}$ supported in $B_{(x, r)}$. We put $\varphi(y)=r^{n} \Phi(x+r y)$ for all $y \in \mathbb{R}^{n}$. Then $\varphi$ is supported in $\mathbb{B}$ and

$$
d \varphi(y)=r^{n+1} \sum_{I, j}\left(\partial_{j} \Phi_{I}\right)(x+r y) e^{j} \wedge e^{I}=r^{n+1} d \Phi(x+r y) .
$$

So, we obtain 


$$
\|d \varphi\|_{L^{q\left(\mathbb{B}, \Lambda^{n-\ell+1}\right)}}=r\|d \Phi\|_{L^{q\left(\mathbb{R}^{n}, \Lambda^{n-\ell+1}\right)}} \leq\left|B_{(x, r)}\right|^{-\frac{1}{q^{\prime}}},
$$

according to the definition of $\Phi \in \overrightarrow{\mathcal{F}}_{x, d}^{q}$. To conclude for the lemma, it is sufficient to find $\psi$ in $W^{1, q}\left(\mathbb{R}^{n}, \Lambda^{n-\ell}\right)$ supported in $\mathbb{B}$ and such that $d \psi=d \varphi$ with

$$
\|\psi\|_{W^{1, q}\left(\mathbb{R}^{n}, \Lambda^{n-\ell}\right)} \leq C\|d \varphi\|_{L^{q\left(\mathbb{B}, \Lambda^{n-\ell+1}\right)}} .
$$

Indeed, if we let $\Psi(y)=\psi_{r}(y-x)$, then $C^{-1} \Phi \in \overrightarrow{\mathcal{F}}_{x}^{q}$, and $d \Psi=d \Phi$, so that $\int f \wedge \Phi=\int f \wedge \Psi$.

So we conclude easily from the following lemma.

Lemma 3.5. Let $1<q<\infty$ and $1 \leq \ell \leq n-1$. Let $\mathbb{B}$ be the unit ball. Let $\varphi \in L^{\infty}\left(\mathbb{R}^{n}, \Lambda^{\ell}\right)$ compactly supported in $\mathbb{B}$ such that $d \varphi$ is in $L^{q}\left(\mathbb{R}^{n}, \Lambda^{\ell+1}\right)$. Then there exists $\psi \in W^{1, q}\left(\mathbb{R}^{n}, \Lambda^{\ell}\right)$ vanishing outside $\mathbb{B}$, such that $d \psi=d \varphi$. Moreover, we can choose $\psi$ such that

$$
\|\psi\|_{W^{1, q}\left(\mathbb{R}^{n}, \Lambda^{\ell}\right)} \leq C\|d \varphi\|_{L_{\left(\mathbb{R}^{n}, \Lambda^{\ell+1}\right)}}
$$

for some uniform constant $C$.

Proof. The existence of a form $\psi \in W_{0}^{1, q}\left(\mathbb{B}, \Lambda^{\ell}\right)$ such that $d \psi=d \varphi$ is given by Theorem 3.3.3 of [Sc]. Moreover, one has the inequality

$$
\|\psi\|_{W^{1, q\left(\mathbb{B}, \Lambda^{\ell}\right)}} \leq C\|d \varphi\|_{L^{q}\left(\mathbb{B}, \Lambda^{\ell+1}\right)} .
$$

Then $\psi$ extends into a form of $W^{1, q}\left(\mathbb{R}^{n}, \Lambda^{\ell}\right)$ when given the value 0 outside the unit ball. We still note $\psi$ the form on $\mathbb{R}^{n}$, which is supported by $\mathbb{B}$.

This allows to conclude for the proof of Lemma 3.4

\section{Wedge PRODUCTS}

We are interested in estimates of wedge products of two differential forms of degree $\ell$ and $n-\ell$ respectively, with $1 \leq \ell \leq n-1$. Recall that, for $f=\sum_{I} f_{I} e^{I} \in \mathcal{C}\left(\mathbb{R}^{n}, \Lambda^{\ell}\right)$ and $g=\sum_{J} g_{J} e^{J} \in \mathcal{C}\left(\mathbb{R}^{n}, \Lambda^{\mathfrak{n}-\ell}\right)$, with $I$ varying among all ordered $\ell$-tuples $1 \leq i_{1}<\ldots<i_{\ell} \leq n$ and $J$ among all ordered $n-\ell$-tuples, we put

$$
f \wedge g=\sum_{I, J}\left(f_{I} \cdot g_{J}\right) e^{I} \wedge e^{J} .
$$

The $n$-form $f \wedge g$ identifies with a function via the Hodge operator. It is clear that the wedge product can also be defined as soon as products are. In particular, it is the case when $f \in L^{p}\left(\mathbb{R}^{n}, \Lambda^{\ell}\right)$ and $g \in L^{q}\left(\mathbb{R}^{n}, \Lambda^{n-\ell}\right)$, with $\frac{1}{p}+\frac{1}{q} \leq 1$. Using the results of [BIJZ] and [BF], it is also the case when one 
of the two forms belongs to the Hardy space $\mathcal{H}^{p}\left(\mathbb{R}^{n}, \Lambda^{\ell}\right)$ while the other one is in the dual space. Moreover, it is proved that

$$
f \wedge g \in L^{1}\left(\mathbb{R}^{n}, \Lambda^{n}\right)+\mathcal{H}_{\omega}^{\Phi}\left(\mathbb{R}^{n}, \Lambda^{n}\right)
$$

if $f \in \mathcal{H}^{1}\left(\mathbb{R}^{n}, \Lambda^{\ell}\right)$ and $g \in \mathfrak{b m o}\left(\mathbb{R}^{n}, \Lambda^{n-\ell}\right)$, while

$$
f \wedge g \in L^{1}\left(\mathbb{R}^{n}, \Lambda^{n}\right)+\mathcal{H}^{p}\left(\mathbb{R}^{n}, \Lambda^{n}\right)
$$

if $p<1, f \in \mathcal{H}^{p}\left(\mathbb{R}^{n}, \Lambda^{\ell}\right)$ and $g \in \Lambda_{n\left(\frac{1}{p}-1\right)}\left(\mathbb{R}^{n}, \Lambda^{n-\ell}\right)$. Here $\mathcal{H}_{\omega}^{\Phi}\left(\mathbb{R}^{n}, \Lambda^{n}\right)$ is the Hardy Orlicz space associated to the function $\Phi(t)=\frac{t}{\log (e+t)}$ and $\omega(x)=$ $(\log (e+|x|))^{-1}$.

We are now interested in improving these estimates when $f$ and $g$ are closed. The div-curl lemma can be generalized to closed forms: this has already been observed by Lou and Mc Intosh in [LM1] when $\frac{1}{p}+\frac{1}{q}=1$. In general, we can state the following.

Theorem 4.1. Let $\frac{n}{n+1}<p \leq 1$ and $1 \leq \ell \leq n-1$. Let $1<q \leq \infty$ be such that $\frac{1}{r}:=\frac{1}{p}+\frac{1}{q} \leq \frac{n+1}{n}$. Then, if $f \in \mathcal{H}_{d}^{p}\left(\mathbb{R}^{n}, \Lambda^{\ell}\right) \cap L^{q^{\prime}}\left(\mathbb{R}^{n}, \Lambda^{\ell}\right)$ and $g \in L^{q}\left(\mathbb{R}^{n}, \Lambda^{n-\ell}\right)$ is such that $d g=0$, then $f \wedge g \in \mathcal{H}_{d}^{r}\left(\mathbb{R}^{n}, \Lambda^{n}\right)$. Moreover, there exists a constant $C$ not depending on $f$ and $g$, such that

$$
\|g \wedge f\|_{\mathcal{H}_{d}^{r}\left(\mathbb{R}^{n}, \Lambda^{n}\right)} \leq C\|g\|_{L^{q}\left(\mathbb{R}^{n}, \Lambda^{n-\ell}\right)}\|f\|_{\mathcal{H}_{d}^{p}\left(\mathbb{R}^{n}, \Lambda^{\ell}\right)} .
$$

Proof. Remark that the forms $f \in \mathcal{H}_{d}^{p}\left(\mathbb{R}^{n}, \Lambda^{\ell}\right) \cap L^{q^{\prime}}\left(\mathbb{R}^{n}, \Lambda^{\ell}\right)$ are dense in $\mathcal{H}_{d}^{p}\left(\mathbb{R}^{n}, \Lambda^{\ell}\right)$ : just take $f * P_{\varepsilon}$, where $P_{t}$ is the Poisson kernel, to approach $f$. Remark also that the assumptions can be made symmetric: just replace $\ell$ by $n-\ell$.

To adapt the proof given in CLMS the main point is given in the next lemma, which has its own interest.

Lemma 4.2. Let $\frac{n}{n+1}<p \leq 1$ and $1 \leq \ell \leq n-1$. Then, for $f \in \mathcal{H}_{d}^{p}\left(\mathbb{R}^{n}, \Lambda^{\ell}\right)$, there exists $h \in L_{d}^{p^{*}}\left(\mathbb{R}^{n}, \Lambda^{\ell-1}\right)$ such that $d h=f$ and $\delta h=0$, with $\frac{1}{p^{*}}=\frac{1}{p}-\frac{1}{n}$. Moreover $h$ is unique up to the addition of a constant form, and there exists some uniform constant $C$ such that

$$
\left\|\mathcal{M}_{S o b}(f)\right\|_{p} \leq C\|f\|_{\mathcal{H}^{p}},
$$

with

$$
\mathcal{M}_{\text {Sob }}(f)(x)=\sup _{t>0} \frac{1}{t|B(x, t)|} \int_{B(x, t)}\left|h(y)-h_{B(x, t)}\right| d y .
$$

Recall that $h_{B(x, t)}$ is the mean of $h$ over the ball $B(x, t)$, which is well defined since $h$ is in $L^{p^{*}}$ and $p^{*}>1$.

Remark that $\mathcal{M}_{S o b}(f)$ is independent on the choice of $h$ since $h$ is unique up to the addition of a constant form. 
Proof. The case $\ell=1$ is Lemma II.2 of [CLMS]. So it is sufficient to consider $\ell>1$. Let us first remark that the uniqueness is direct, since in $L^{p^{*}}\left(\mathbb{R}^{n}, \Lambda^{\ell-1}\right)$ only constants are $d$-closed and $\delta$-closed. Assume that $h$ is a solution. Then all the derivatives $\partial_{j} h_{I}$ are in the Hardy space $\mathcal{H}^{p}\left(\mathbb{R}^{n}\right)$. Indeed, we use the fact that, by definition of $\Delta_{\ell-1}$, we have the identities

$$
\Delta_{\ell-1} h=\delta f, \quad \partial_{j} h=\left(\partial_{j}\left(-\Delta_{\ell-1}\right)^{-1 / 2}\right)\left(\left(-\Delta_{\ell-1}\right)^{-1 / 2} \delta\right) f .
$$

But both operators arising in the last expression, that is $\partial_{j}\left(-\Delta_{\ell-1}\right)^{-1 / 2}$ and $\left(-\Delta_{\ell-1}\right)^{-1 / 2} \delta$, are linear combinations of Riesz transforms and preserve Hardy spaces. Indeed, since $\Delta_{\ell-1}$ is given by the Laplacian, coefficients by coefficients, the same is valid for all its powers. Furthermore, we have

$$
\|\nabla h\|_{\mathcal{H}^{p}} \leq C\|f\|_{\mathcal{H}^{p}} .
$$

Conversely, given $f$, we can use these formulas to fix the values of $\partial_{j} h_{I}$ in the Hardy space $\mathcal{H}^{p}\left(\mathbb{R}^{n}\right)$. Using this lemma for one-forms, we know the existence of $h_{I} \in L^{p^{*}}\left(\mathbb{R}^{n}\right)$ having these functions as derivatives. It is elementary to see that the form $h=\sum h_{I} e^{I}$ is such that $d h=f$ and $\delta h=0$, using commutation properties of the operators. Finally, we write (50) for each $f_{j}=\partial_{j} h_{I}$ to obtain the inequality for $f$.

It is elementary to adapt the rest of the proof of Theorem II.3 in [CLMS, once the lemma has been settled, and we leave it to the reader. So this gives the proof of Theorem 4.1. Remark that $f \wedge g$ can be defined in the distribution sense without the additional assumption that $g \in L^{q^{\prime}}\left(\mathbb{R}^{n}, \Lambda^{\ell}\right)$ : we pose $f \wedge g=$ $d(h \wedge g)$, with $h$ given by Lemma 4.2. Indeed, $h \wedge g$ is in the Lebesgue space $L^{s}$, with $s>1$ given by $\frac{1}{s}=\frac{1}{r}-\frac{1}{n}$. So its exterior derivative is well defined as a distribution.

Following the ideas of [ART, let us sketch another proof for the endpoint $q=\infty$.

Proof for the endpoint. Let $f \in \mathcal{H}_{d}^{p}\left(\mathbb{R}^{n}, \Lambda^{\ell}\right)$ and $g \in L^{\infty}\left(\mathbb{R}^{n}, \Lambda^{n-\ell}\right)$ such that $d g=0$. We want to prove that

$$
\mathcal{M}(f \wedge g)(x) \leq C\|g\|_{L^{\infty}\left(\mathbb{R}^{n}, \Lambda^{n-\ell}\right)} \overrightarrow{\mathcal{M}}_{\infty, d} f(x),
$$

from which we conclude directly by using Lemma 3.3 and Lemma 3.4. By linearity we can assume that $\|g\|_{\infty}=1$. In order to estimate $\mathcal{M}(f \wedge g)(x)$, we have to consider

$$
\int \varphi_{x, t} f \wedge g, \quad \varphi_{x, t}(y)=t^{-n} \varphi((x-y) / t) .
$$

Here $\varphi$ is chosen smooth and supported in the unit ball as in (11). It is sufficient to prove the existence of some uniform constant $c$ such that $c \varphi_{x, t} g$ 
belongs to $\overrightarrow{\mathcal{F}}_{x, d}^{\infty}$. This follows from the inequality

$$
\left\|\varphi_{x, t}\right\|_{\infty}+t\left\|d \varphi_{x, t}\right\|_{\infty} \leq\left|B_{(x, t)}\right|^{-1} .
$$

Indeed, since $g$ is closed, we have the equality $d\left(\varphi_{x, t} g\right)=d \varphi_{x, t} \wedge g$ and the uniform norm of a wedge product is bounded by the product of norms.

This finishes the proof.

\section{BMO ESTIMATES}

Let us first recall some facts on $B M O\left(\mathbb{R}^{n}\right)$ and weighted Hardy-Orlicz spaces.

Given a continuous function $\mathcal{P}:[0, \infty) \rightarrow[0, \infty)$ increasing from 0 to $\infty$ (but not necessarily convex, $\mathcal{P}$ is called the Orlicz function), and given a positive measurable function $\omega$, the weighted Orlicz space $L_{\omega}^{\mathcal{P}}\left(\mathbb{R}^{n}\right)$ consists in the set of functions $f$ such that

$$
\|f\|_{L_{\omega}^{\mathcal{P}}\left(\mathbb{R}^{n}\right)}:=\inf \left\{k>0: \int_{\mathbb{R}^{n}} \mathcal{P}\left(k^{-1}|f|\right) \omega(x) d x \leq 1\right\}
$$

is finite. The Hardy Orlicz space $\mathcal{H}_{\omega}^{\mathcal{P}}\left(\mathbb{R}^{n}\right)$ (resp. the local Hardy Orlicz space $\mathfrak{h}_{\omega}^{\mathcal{P}}\left(\mathbb{R}^{n}\right)$ is the space of tempered distributions $f$ such that $\mathcal{M} f\left(\right.$ resp. $\left.\mathcal{M}^{(1)} f\right)$ belongs to $L_{\omega}^{\mathcal{P}}\left(\mathbb{R}^{n}\right)$. We will consider here the Orlicz space associated to the function $\Phi(t)=\frac{t}{\log (e+t)}$ and the weight $(\log (e+|x|))^{-1}$, as mentioned in the introduction. The space $L_{\omega}^{\Phi}\left(\mathbb{R}^{n}\right)$ is not a normed space. Let us state the following properties of the function $\Phi$ and $L_{\omega}^{\Phi}$.

$$
\begin{aligned}
\Phi(s+t) & \leq \Phi(s)+\Phi(t), & & \text { for all } s, t>0 . \\
\Phi(s t) & \geq \Phi(s) \Phi(t), & & \text { for all } s, t>0 . \\
\Phi(s t) & \leq s+e^{t}-1 & & \text { for all } s, t>0 . \\
\Phi(s t) & \leq \log (e+d)\left(s+\frac{1}{d}\left(e^{t}-1\right)\right) & & \text { for all } s, t, d>0 .
\end{aligned}
$$

The two first inequalities are elementary. The third one is given in [BIJZ]. For $d>0$, we write that $\Phi(d) \Phi(s t) \leq \Phi((s d) t)$, and use the previous one with $s d$ in place of $s$.

Next, by using the fact that $\Phi(4)>2$ and (53) we obtain the inequality

$$
\Phi\left(\frac{s+t}{4}\right) \leq \frac{\Phi(s)+\Phi(t)}{2}
$$

from which we conclude that

$$
\|f+g\|_{L_{\omega}^{\Phi}\left(\mathbb{R}^{n}\right)} \leq 4\|f\|_{L_{\omega}^{\Phi}\left(\mathbb{R}^{n}\right)}+4\|g\|_{L_{\omega}^{\Phi}\left(\mathbb{R}^{n}\right)} .
$$

We will also need the fact that products of integrable functions with functions in the exponential class are in $L^{\Phi}$. More precisely, we will use the following lemma, for $B$ a ball with radius 1 . It is a direct consequence of (55). 
Lemma 5.1. For $c>0$ given, there exists $C$ such that, for $d>0$,

$$
\int_{B} \Phi(|f g|) \frac{d x}{\log (e+d)} \leq C \int_{B}|f| d x+\frac{C}{d} \int_{B}\left(e^{c|g|}-1\right) d x .
$$

Our main theorem is the following.

Theorem 5.2. Let $f \in \mathcal{H}_{d}^{1}\left(\mathbb{R}^{n}, \Lambda^{\ell}\right)$ and $g \in \mathfrak{b m o}\left(\mathbb{R}^{n}, \Lambda^{n-\ell}\right)$ such that $d g=0$, then the product $f \wedge g$ is in $\mathcal{H}_{\omega}^{\Phi}\left(\mathbb{R}^{n}, \Lambda^{n}\right)$. Moreover, there exists a uniform constant $C$ such that

$$
\|f \wedge g\|_{\mathcal{H}_{\omega}^{\Phi}\left(\mathbb{R}^{n}, \Lambda^{n}\right)} \leq C\|f\|_{\mathcal{H}^{1}\left(\mathbb{R}^{n}, \Lambda^{\ell}\right)}\|g\|_{\mathfrak{b m o}\left(\mathbb{R}^{n}, \Lambda^{n-\ell}\right)} .
$$

Proof. Recall that the wedge product $f \wedge g$ is well defined in the distribution sense for $f \in \mathcal{H}_{d}^{1}\left(\mathbb{R}^{n}, \Lambda^{\ell}\right)$ and $g \in \mathfrak{b} \mathfrak{m o}\left(\mathbb{R}^{n}, \Lambda^{n-\ell}\right)$. It is sufficient to have an a priori estimate for $g$ bounded, which we assume from now on. We also assume that $\|g\|_{\mathfrak{b m o}\left(\mathbb{R}^{n}, \Lambda^{n-\ell}\right)}=1$ and $\|f\|_{\mathcal{H}^{1}\left(\mathbb{R}^{n}, \Lambda^{\ell}\right)}=1$. Then, for $x \in \mathbb{R}^{n}$ and $\varphi \in \mathcal{F}_{x}^{\infty}$ supported in $B(x, r)$, we have

$$
\left|\int(f \wedge g) \varphi\right| \leq\left|\int f \wedge\left(g-g_{B(x, r)}\right) \varphi\right|+\left|\int f \wedge\left(g_{B(x, r)} \varphi\right)\right|,
$$

where

$$
g_{B(x, r)}=\sum_{I}\left(g_{I}\right)_{B(x, r)} e^{I} \text { if } g=\sum_{I} g_{I} e^{I} .
$$

Let us first evaluate the second term of the sum (59). We have

$$
\begin{aligned}
\left|\int f \wedge\left(g_{B(x, r)} \varphi\right)\right| & =\left|\sum_{I}\left(g_{I}\right)_{B(x, r)} \int f \wedge \varphi e^{I}\right| \\
& \leq\left(1+\mathfrak{M}^{(1)} g(x)\right) \overrightarrow{\mathcal{M}}_{\infty}(f)(x)
\end{aligned}
$$

where $\mathfrak{M}^{(1)} g(x)=\sum_{I} \mathfrak{M}^{(1)} g_{I}(x) e^{I}$, and for $h \in L_{l o c}^{1}\left(\mathbb{R}^{n}\right)$ a scalar valued function,

$$
\mathfrak{M}^{(1)} h(x)=\sup \left\{\frac{1}{|B|} \int_{B}|h(y)| d y, x \in B \text { and }|B|<1\right\} .
$$

Indeed, when $r \geq 1$, the mean of $g$ is, by definition of $\mathfrak{b m o}$, bounded by $\|g\|_{\mathfrak{b m o}\left(\mathbb{R}^{n}, \Lambda^{n-\ell}\right)}=1$, while, for $r<1$, it is bounded by the maximal function related to small balls. For the first term, we proceed in the same way as for bounded $g$. By John-Nirenberg Inequality, the form $\left(g-g_{B(x, r)}\right) \varphi$ satisfies Condition $(* *)$ for all $q>1$ up to some uniform constant $C$. So, according to Lemma 3.4, for $q$ large enough,

$$
\left|\int f \wedge\left(g-g_{B(x, r)}\right) \varphi\right| \leq C \overrightarrow{\mathcal{M}}_{q} f(x) .
$$


Taking in (61) and (63) the supremum over all $\varphi \in \mathcal{F}_{x}^{\infty}$ yields

$$
\mathcal{M}_{\infty}(f \wedge g) \leq C\left(\overrightarrow{\mathcal{M}}_{q}(f)+\mathfrak{M}^{(1)}(g) \overrightarrow{\mathcal{M}}_{\infty}(f)\right)
$$

The first term is in $L^{1}\left(\mathbb{R}^{n}\right)$ under the assumption that $f \in \mathcal{H}_{d}^{1}\left(\mathbb{R}^{n}, \Lambda^{\ell}\right)$ according to Lemma 3.3. It remains to prove that the second term is in $L_{\omega}^{\Phi}\left(\mathbb{R}^{n}\right)$ to conclude the proof of our theorem. Roughly speaking, this is the consequence of the fact that the product of an integrable function with a function in the exponential class is in the Orlicz space $L^{\Phi}$. Indeed, we recall that, by John-Nirenberg's Inequality, there exists $c>$ such that, for each ball $B$,

$$
\int_{B} e^{c\left|g-g_{B}\right|} d x \leq C
$$

The following lemma allows to have the same kind of estimate for $\mathfrak{M}^{(1)}(g)$ in place of $g$.

Lemma 5.3. Let $c>0$ fixed. Then there exists some uniform constant $C$ such that

$$
\int_{B} e^{\frac{c}{2}\left|\mathfrak{M}^{(1)}(g)\right|} \leq C \int_{3 B} e^{c|g|} d x
$$

for every ball $B$ with radius 1 . The ball $3 B$ is the ball with same center and radius 3 .

Proof. We have

$$
\int_{B} e^{\frac{c}{2}\left|\mathfrak{M}^{(1)}(g)(x)\right|} d x \leq e^{c}|B|+\int_{1}^{\infty} e^{\frac{c s}{2}}\left|\left\{x \in B: \mathfrak{M}^{(1)}(g)>s\right\}\right| d s .
$$

Let $g_{1}=g \chi_{3 B}$, where $3 B$ is the ball having same center as $B$, but with radius 3 times the one of $B$ and $\chi_{3 B}$ is the characteristic function of $3 B$. It is easy to see that for all $s>0$, we have

$$
\left\{x \in B: \mathfrak{M}^{(1)} g(x)>s\right\} \subset\left\{x \in \mathbb{R}^{n}: \mathfrak{M} g_{1}(x)>s\right\} .
$$

Thus from the above inclusion and the weak type $(1,1)$ boundedness of the Hardy-littlewood maximal function, we have

$$
\left|\left\{x \in B: \mathfrak{M}^{(1)}(g)>s\right\}\right| \leq\left|\left\{x \in \mathbb{R}^{n}: \mathfrak{M} g_{1}(x)>s\right\}\right| \leq \frac{C}{s} \int_{\left\{\left|g_{1}\right|>\frac{s}{2}\right\}}\left|g_{1}(x)\right| d x
$$


with $C$ independent of $B$ and $g$, so that the integral in the second member becomes

$$
\begin{aligned}
\int_{1}^{\infty} e^{\frac{c s}{2}}\left|\left\{x \in B: \mathfrak{M}^{(1)}(g)>s\right\}\right| d s & \leq C \int_{1}^{\infty} \frac{e^{\frac{c s}{2}}}{s}\left(\int_{\left\{|g|>\frac{s}{2}\right\} \cap 3 B}|g(x)| d x\right) d s \\
& \leq C^{\prime} \int_{3 B} e^{c|g(x)|} d x
\end{aligned}
$$

by using Fubini's Theorem. We conclude for the lemma.

Let us come back to the proof of Theorem 5.2, We write $\mathbb{R}^{n}$ as the almost disjoint union of balls $B_{j}=: \mathbb{B}+j$, with $j \in \mathbb{Z}^{n}$, with $\mathbb{B}$ the unit ball centered at 0 . We make use of Lemma 5.1 on each of these balls, with $d:=d_{j}:=(1+|j|)^{-N}$, with $N$ large enough so that $\sum d_{j}^{-1}<\infty$ while $\omega(x) \simeq\left(\log d_{j}\right)^{-1}$ for $x \in B_{j}$. We recall that by assumption $\left|g_{3 B_{j}}\right| \leq C$, since $\|g\|_{\mathfrak{b m o}\left(\mathbb{R}^{n}, \Lambda^{n-\ell}\right)}=1$. So

$$
\int_{3 B_{j}} e^{c|g(x)|} d x \leq C
$$

We finally have

$$
\int_{\mathbb{R}^{n}} \Phi\left(\mathfrak{M}^{(1)}(g)(x) \overrightarrow{\mathcal{M}}_{\infty}(f)(x)\right) \omega(x) d x \leq C \sum_{j \in \mathbb{Z}^{n}} \int_{B_{j}}|f| d x,
$$

from which we conclude that $\mathfrak{M}^{(1)}(g) \overrightarrow{\mathcal{M}}_{\infty}(f)$ has a bounded norm in $L_{\omega}^{\Phi}\left(\mathbb{R}^{n}\right)$ because of the finite overlapping of balls $B_{j}$. By finite additivity (56) we have

$$
\|\mathcal{M}(f \wedge g)\|_{L_{\omega}^{\Phi}\left(\mathbb{R}^{n}\right)} \leq 4 C\left\|\overrightarrow{\mathcal{M}}_{q} f\right\|_{L_{\omega}^{\Phi}\left(\mathbb{R}^{n}\right)}+4 C\left\|\mathfrak{M}^{(1)}(g) \overrightarrow{\mathcal{M}}_{\infty}(f)\right\|_{L_{\omega}^{\Phi}\left(\mathbb{R}^{n}\right)}
$$

so that

$$
\|f \wedge g\|_{\mathcal{H}_{\omega}^{\Phi}\left(\mathbb{R}^{n}, \Lambda^{n}\right)} \leq C
$$

for $\|f\|_{\mathcal{H}^{1}\left(\mathbb{R}^{n}, \Lambda^{\ell}\right)}=1$.

We have as well the following theorem.

Theorem 5.4. Let $f \in \mathfrak{h}_{d}^{1}\left(\mathbb{R}^{n}, \Lambda^{\ell}\right)$ and $g \in B M O\left(\mathbb{R}^{n}, \Lambda^{n-\ell}\right)$ such that $d g=0$, then the product $f \wedge g$ is in $\mathfrak{h}_{\omega}^{\Phi}\left(\mathbb{R}^{n}, \Lambda^{n}\right)$. Moreover, there exists a uniform constant $C$ such that

$$
\|f \wedge g\|_{\mathfrak{h}_{\omega}^{\Phi}\left(\mathbb{R}^{n}, \Lambda^{n}\right)} \leq C\|f\|_{\mathfrak{h}^{1}\left(\mathbb{R}^{n}, \Lambda^{\ell}\right)}\|g\|_{B M O\left(\mathbb{R}^{n}, \Lambda^{n-\ell}\right)} .
$$


The key point is that again we only have to make use of $\mathfrak{M}^{(1)} g$ and not of $\mathfrak{M} g$. The only difference in the proof is the replacement of (67) by

$$
\int_{3 B_{j}} e^{c|g(x)|} d x \leq C(1+|j|)^{M}
$$

for some $M>0$ : use the well-known fact that $g_{3 B_{j}} \leq C \log (1+|j|)$.

The generalization of Theorem 5.2 to $\mathcal{H}_{\omega}^{p}$ for $p<1$ is direct from (64). Then the product $f \wedge g$ belongs to $\mathcal{H}_{\omega}^{\Phi_{p}}$, with $\Phi_{p}(t)=\left(\frac{t}{\log (e+t)}\right)^{p}$. Remark that, in this case, the product of a function in $\mathfrak{b m o}$ and a function in $\mathcal{H}^{p}$ does not make sense as a distribution in general. But we can establish as above an a priori estimate, which allows to give a meaning to the wedge product of two closed forms.

\section{REFERENCES}

[ART] P. Auscher, E. Russ and P. Tchamitchian, Hardy sobolev spaces on strongly Lipschitz domains of $\mathbb{R}^{n}$, J. Funct. Anal. 218 (2005), 54-109.

[BF] A. Bonami and J. Feuto, Products of functions in Hardy and Lipschitz or BMO spaces, Preprint, arXiv:0806.485.

[BG] A. Bonami and S. Grellier, Decomposition Theorems for Hardy-Orlicz spaces and weak factorization. Preprint arXiv:0902.2138.

[BIJZ] A. Bonami, T. Iwaniec, P. Jones and M. Zinsmeister, On the product of Functions in BMO and $\mathcal{H}^{1}$, Ann. Inst. Fourier, Grenoble 57, 5 (2007) 1405-1439.

[CLMS] R. Coifman, P.L. Lions, Y. Meyer and S. Semmes, Compensated compactness and Hardy spaces, J. Math. Pures Appl. 72 (1993), 247-286.

[CMS1] R. Coifman, Y. Meyer and E. M. Stein, Some new function spaces and their application to harmonic analysis, J. Funct. Anal. 62 (1985), 304-335.

[CMS2] R. Coifman and G. Weiss, Extensions of Hardy spaces and their use in analysis, Bull. Amer. J. Math. 83 (1977), 569-645.

[D] G. Dafni, Nonhomogeneous div-curl lemmas and local Hardy spaces, Adv. Differential Equations 10 (2005), 505-526.

[FS] G.B. Folland and E.M. Stein, Hardy spaces on homogeneous groups, Mathematical Notes, 28. Princeton University Press, Princeton, N.J.; University of Tokyo Press, Tokyo, 1982.

[G] D. Golberg, A local version of Hardy spaces, Duke J. Math. 46 (1979), 27-42.

[ISS] T. Iwaniec, C. Scott and B. Stroffolini Nonlinear Hodge theory on manifolds with boundary, Ann. Mat. Pura Appl. (4) 177 (1999), 37-115.

[LM1] Z. Lou and A. M ${ }^{c}$ Intosh, Hardy space of exact forms on $\mathbb{R}^{N}$, Trans. Amer. Math. Soc. $357\left(N^{0} 4\right)$ (2005), 1469-1496.

[LM2] Z. Lou, A and McIntosh, Hardy space of exact forms on lipschitz domains in $\mathbb{R}^{N}$, Indiana Univ. Math. J. 53 (2004), 583-611.

[M] F. Murat, Compacité par compensation Ann. Sc. Norm. Sup. Pisa 5 (1978) 489-507.

[Sc] G. Schwartz, Hodge decomposition-A method for solving boundary value problems, Lecture Notes in Mathematics vol. 1607, Springer-Verlag, Berlin Heidelberg, 1995.

[St] E. M. Stein, Harmonic analysis, real-variable methods, orthogonality, and oscillatory integrals, Princeton Math. Series 43, Princeton University Press, Princeton 1993. 
[Ta] L. Tartar, Compensated compactness and applications to partial differential equations, Nonlinear analysis and mechanics: Heriot-Watt Symposium, Vol. IV, Res. Notes in Math., 39, Pitman, Boston, Mass.-London, (1979) 136-212.

MAPMO-UMR 6628, DÉPartement de Mathématiques, Université D’Orleans, 45067 Orléans Cedex 2, France

E-mail address: Aline.Bonami@univ-orleans.fr

Laboratoire de Mathématiques Fondamentales, UFR Mathématiques et InFormatique, Université de Cocody, 22 B.P 1194 Abidjan 22. Côte D'Ivoire

E-mail address: justfeuto@yahoo.fr

MAPMO-UMR 6628, Département de Mathématiques, Université D'Orleans, 45067 Orléans Cedex 2, France

E-mail address: Sandrine.Grellier@univ-orleans.fr 